Article

\title{
High-Sensitive TM Modes in Photonic Crystal Nanobeam Cavity with Horizontal Air Gap for Refractive Index Sensing
}

\author{
Jin-Kyu Yang *D, Chae-Young Kim and Minji Lee \\ Department of Optical Engineering, Kongju National University, Cheonan 31080, Korea; \\ codud3321@naver.com (C.-Y.K.); mj19659@naver.com (M.L.) \\ * Correspondence: jinkyuyang@kongju.ac.kr; Tel.: +82-41-521-9445
}

Received: 26 February 2019; Accepted: 4 March 2019; Published: 7 March 2019

check for updates

\begin{abstract}
We propose a new type of refractive index sensing based on the transverse magnetic (TM) modes in the photonic crystal (PhC) nanobeam (NB) cavity with a horizontal air gap. The electric field of the resonant TM mode is strongly confined within the horizontal air gap present at the PhC NB cavity. In order to increase the quality $(Q)$ factor and the sensitivity $(S)$ of the refractive index change in the air simultaneously, the cavity structure is fully optimized. Because of the trade-off between the Q-factor and $S$ of the TM mode in the PhC NB cavity with an air gap, there is an optimal thickness of the air gap in the dielectric slot. From the numerical simulation results, $S$ can exceed $1000 \mathrm{~nm} / \mathrm{RIU}$ with $Q>40,000$. When the dielectric slot becomes thin, $S$ could be higher than $1200 \mathrm{~nm} / \mathrm{RIU}$. For practical applications, we suggest an Si-based $\mathrm{PhC} \mathrm{NB}$ cavity with a horizontal $\mathrm{SiO}_{2}$ slot structure which can also provide high $S$ with a high Q-factor after a very fine selective wet etching process. This new type of TM resonant mode in the PhC NB cavity can be an ideal platform for compact sensors in photonic integrated circuits for TM waveguide systems.
\end{abstract}

Keywords: photonic crystals; refractive index sensing; TM mode

\section{Introduction}

Photonic crystals (PhCs) have been widely studied for their ability to prohibit light propagation due to their photonic bandgap (PBG) [1,2]. In particular, PhC cavity structures have attracted many researchers due to their small modal volume $(\mathrm{V})$ and high quality $(\mathrm{Q})$ factor with help of the rapid development of nanotechnology processes such as lithography and dry etching [3-5]. One of the possible applications of $\mathrm{PhC}$ cavity structures is optical sensing based on wavelength shift due to environmental changes such as $\mathrm{pH}$ changes, chemical changes, and strain changes [6-8].

Recently, PhC nanobeam (NB) structures have been proposed as new types of optical integrated circuits because of structural simplicity to achieve an ultrahigh $\mathrm{Q} / \mathrm{V}$ ratio and direct coupling to optical waveguides [9-11]. Furthermore, PhC NB cavity structures are ideally suitable for ultrasensitive optical sensing with an extremely small footprint because of the deterministic wavelength-scale design $[12,13]$. Moreover, nanometer-wide air gap geometry surrounded by a high-index region was proposed to enhance and confine the light [14]. As the electric field could be tightly concentrated in the air void by large discontinuity at the high-index-contrast interfaces, the $\mathrm{Q} / \mathrm{V}$ ratio and optical sensitivity of the resonant mode in PhC cavity structures with an air gap could increase dramatically $[15,16]$. However, by introducing the air gap geometry in the PhC cavity, the Q-factor decreased due to a high scattering loss. Consequently, the deterministic PhC NB cavity with an air gap was proposed to achieve a high coupling efficiency to the waveguide with a high $Q$ factor $[17,18]$. The $Q$-factor of the resonant cavity mode in PhC NB structures could be increased easily by adding more air holes outside the cavity region to reduce the loss along the waveguide. 
It was also reported that the light could be localized strongly with low index materials in a horizontal slot region [19]. In particular, the scattering loss in the horizontal slot region is smaller than that in the vertical slot due to the smooth interface formed by the simple selective wet etching or oxidation process. Additionally, the horizontal air gap can be made ultrathin by controlling the deposition process, which can enhance the light-matter interaction [20,21]. However, the light localization in the horizontal slot is found in the transverse magnetic (TM) mode among the guided modes in the slab where there is no PBG $[19,20]$. In contrast, the PhC NB structure can have the PBG in both transverse electric (TE) and TM modes [22]. There are several reports related to the horizontally slotted PhC NB cavity; however, there was no systematical investigation of PhC NB cavities for gas sensing [23,24]. In this paper, we numerically investigate the TM mode in a PhC NB cavity with a horizontal air gap from the origin of the PBG to the whole transmission response by the three-dimensional (3-D) plane wave expansion method and the finite-difference time-domain (FDTD) method. Because of the intrinsic trade-off between the Q-factor and the sensitivity of the TM mode in the PhC NB cavity with an air gap, there is an optimal thickness of the air gap in the dielectric slot. The optimized PhC NB cavity is ultrasensitive to a refractive index change of approximately $1020 \mathrm{~nm} /$ RIU with $\mathrm{Q}>$ 40,000. When the dielectric slot becomes thin, the sensitivity can exceed $1200 \mathrm{~nm} / \mathrm{RIU}$. For practical applications, we suggest and Si-based PhC NB cavity with a horizontal $\mathrm{SiO}_{2}$ slot structure. After the very fine selective etching of $\mathrm{SiO}_{2}$, the $\mathrm{PhC} \mathrm{NB}$ cavity with the remaining $\mathrm{SiO}_{2}$ can be mechanically stable and provide high sensitivity with a high Q-factor. The PhC NB cavity with the horizontal air gap can be considered promising for label-free compact optical sensors in photonic integrated circuits for TM waveguide systems.

\section{Methods and Materials}

\subsection{Photonic Band Structure of TM Modes in PhC NB Structure with a Horizontal Air Gap}

Firstly, the photonic band diagram of the TM mode in the PhC NB structure with a horizontal air gap was numerically calculated using the plane wave expansion method [25]. In this study, a free-standing silicon $(n(\mathrm{Si})=3.4)$ waveguide was used because it exhibited no optical absorption and a high refractive index of $\mathrm{Si}$ at the telecommunication wavelength, $1.55 \mu \mathrm{m}$. The upper inset of Figure 1a shows the schematic of the PhC NB structure in which the circular holes were perforated periodically. To carry out the simulation, the width $(w)$, thickness $(t)$, and air gap $\left(t_{g}\right)$ of the Si waveguide were set as $0.95 a, 1.2 a$, and $0.15 a$, respectively, where $a$ is the lattice constant of the air holes. Figure 1a shows the photonic band diagram of the TM modes with $r / a=0.45$ (the solid lines) and $r / a=0.40$ (the dashed lines). The solid and dashed red lines indicate the first TM photonic bands at $r / a=0.45$ and $r / a=0.40$, respectively. As shown in the lower inset of Figure 1a, the first TM mode corresponds to the air gap mode because of the strongly localized electric field distribution at the air region. It can be observed that the normalized frequency of the first TM mode with $r / a=0.45$ at the band edge (wavevector, $k=\pi / a)$ is in the PBG of the TM mode with $r / a=0.40$.

To confine the light in the cavity of PhCs, the resonant frequency of the cavity mode should lie inside the frequency range of the PBG. Figure $1 \mathrm{~b}$ shows the frequencies of the band edge modes as a function of $r / a$. It can be observed that the frequencies of the TM band edge modes decrease as $r / a$ increases. Therefore, to confine the light using the air gap mode, the surrounded air hole should be smaller than the air hole in the cavity region. In this paper, we used $r / a=0.45$ at the center of the PhC NB cavity which was surrounded by air holes with $r / a=0.405$ as the PBG mirror. 
(a)

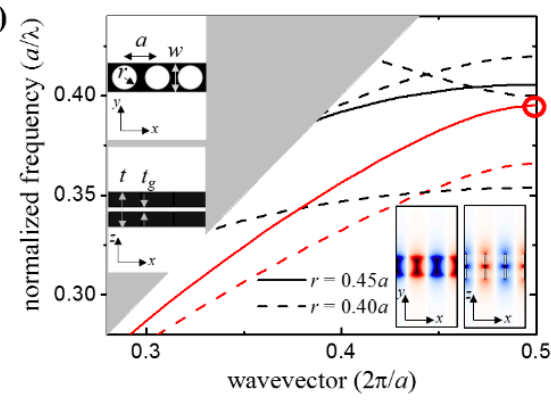

(b)

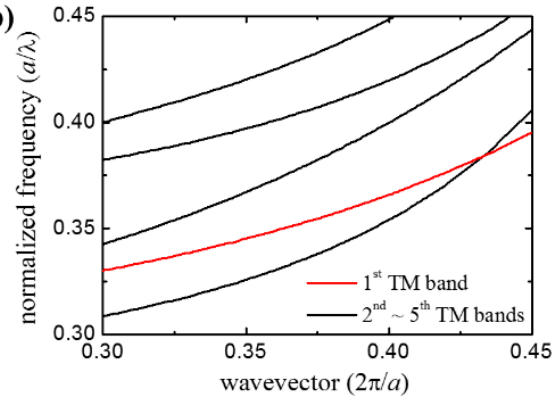

Figure 1. (a) Photonic band diagram of the transverse magnetic (TM) modes at the photonic crystals $(\mathrm{PhC})$ nanobeam $(\mathrm{NB})$ with a horizontal air gap. The solid (or dashed) lines correspond to the radius of the air hole at $r / a=0.45$ (or 0.40 ). The left insets show the top and side views of the PhC NB with the air gap. The right insets are the cut-views of the electric field distribution of the first TM mode and the air gap mode at the band edge marked with the red circle. (b) Normalized frequencies of the TM band edge mode as a function of the radius of air holes. The red line is the band edge frequency of the air gap mode.

\subsection{Optimization of Waveguide Geometry of PhC NB Cavity Structure with a Horizontal Air Gap}

The PhC NB cavity with the horizontal air gap was designed for optimal refractive index sensing using the FDTD method. Figure 2a shows the schematics of the proposed PhC NB cavity structure with a horizontal air gap. According to the previous photonic band calculation, the radius of the air hole, $r_{0}$, at the center is set as $0.45 a$. The radius of the surrounded air hole is gradually reduced to $r_{b m}$, which represents the air holes acting as a PBG mirror for the cavity mode. In this simulation, three air holes with radius $r_{b m}$ are used as a PBG mirror at each waveguide end. It is worth noting that in order to achieve a high Q-factor and sensitivity simultaneously, the radius of the air holes is reduced in a quadratic manner because the dispersion curve of the air gap mode is parabolic, as discussed later in detail [9]. The radius of the $i$-th air hole from the center is set as:

$$
r_{i}=r_{0}-i^{2} \Delta r / m^{2}
$$

where $m$ is the total number of gradually reduced air holes. Here, $m=9$ and the variation in the size of air holes, $\Delta r\left(=r_{0}-r_{b m}\right)$, is set as $0.10 r_{0}$. All other structural parameters are the same as the previous band diagram calculation. Figure $2 \mathrm{~b}$ shows the cut-views of the electric field distribution, $E_{z}$, of the air gap cavity mode along the center plane of the air gap when the width and thickness of the air gap are $w=0.95 a$ and $t_{\mathrm{g}}=0.15 a$, respectively. According to the $x-z$ cut-view, the electric field of the cavity mode is strongly confined in the air gap region, which makes very sensitized to the refractive index change to the environment.

For practical applications of optical sensing, it is necessary to achieve not only high sensitivity but also a high Q-factor. In the first optimization step, we investigated the effect of the width of the $\mathrm{Si}$ waveguide on both sensitivity and Q-factor. The sensitivity, $S$, was defined as follows:

$$
S=\Delta \lambda_{\text {res }} / \Delta n
$$

where $\Delta \lambda_{\text {res }}$ is the peak wavelength shift of the cavity mode due to the refractive index change of the environment, $\Delta n$. Here, we set the range of the refractive index change as 1.000 to 1.001 considering the gas sensing. Figure $2 \mathrm{c}$ shows the $\mathrm{Q}$-factor and $S$ as functions of the width of the $\mathrm{Si}$ waveguide. As the width of the waveguide increases, both the Q-factor and $S$ decrease. The reduction of $S$ and the Q-factor at greater widths is caused by an increase in the electric field in the dielectrics and an increase in waveguide loss, respectively. In contrast, as the thickness of the air gap increases, the $\mathrm{Q}$-factor decreases but $S$ increases. The enhancement of $S$ and reduction of the Q-factor is caused by 
the increase in the air portion and the weak PBG effect, respectively. So, there is a trade-off in order to obtain both a high Q-factor and $S$. Here, we set $t$ and $t_{\mathrm{g}}$ as $1.2 a$ and $0.15 a$, respectively.

(a)

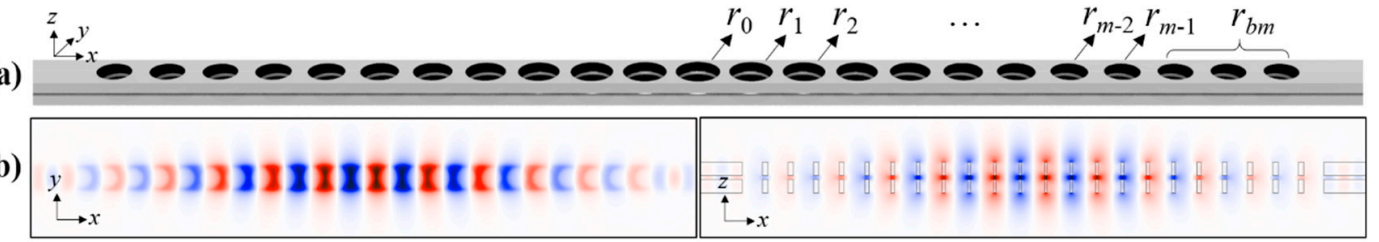

(c)

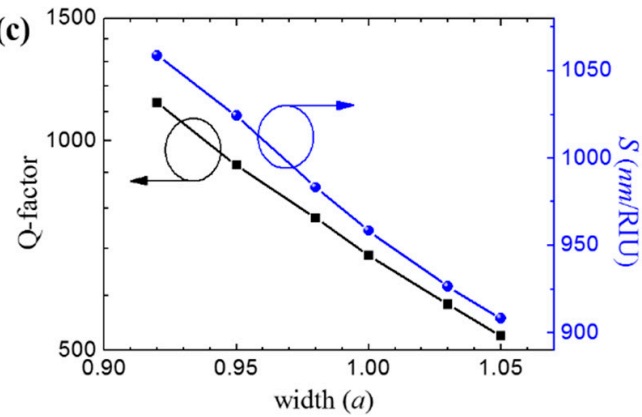

(d)

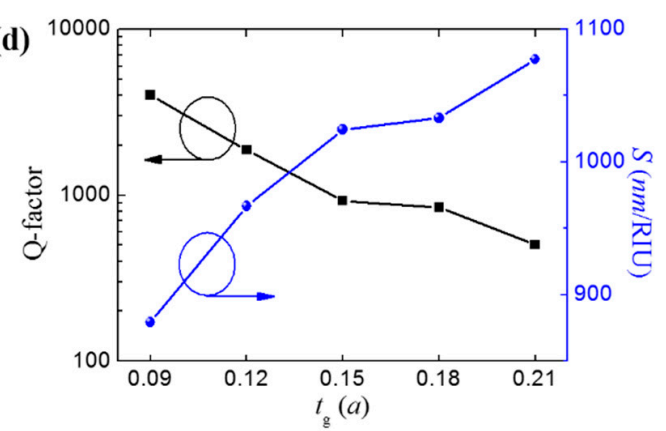

Figure 2. Optimization of the waveguide structure of a PhC NB cavity for refractive index sensing. (a) Schematics of the PhC NB cavity structure with a horizontal air gap; (b) $x-y$ and $x-z$ cut-views of $E_{z}$ field distribution of the air gap cavity mode when width $w=0.95 a$, thickness of air gap $t_{g}=0.15 a$, and size variation of air holes $\Delta r / r_{0}=0.10$; Q-factor (black square) and sensitivity $S$ (nm/RIU) (blue circle) as a function of (c) width $(w)$ of the PhC NB and (d) thickness $\left(t_{\mathrm{g}}\right)$ of the air gap.

In the second optimization step for a high Q-factor and $S$, we investigated the modulation function to reduce air holes from the center to the mirror holes in the PhC NB cavity. Figure 3a shows the Q-factor and $S$ of the air gap cavity mode as a function of the variation of air holes. Here, we employed three different modulation functions; linear, quadratic, and cubic functions. The cavity mode of cubic modulation has a slightly higher $S$ than that of quadratic modulation because the air hole near the center is slightly larger. However, the Q-factor of the cavity mode of quadratic modulation is $8 \%$ higher than that of cubic modulation due to a relatively strong PBG effect. Next, we optimized the variation of the air hole to achieve the maximal Q-factor. Figure 3b shows the Q-factor and $S$ as functions of the variation in the size of air holes. $Q$ achieved a maximum value of approximately 970 at $\Delta r=0.12 r_{0}$, which is limited by the waveguide loss. To understand the optimal condition of waveguide loss, mirror strength $(\gamma)$, as shown in the inset graph of Figure 3b, was calculated using the following equation $[10,18]$ :

$$
\gamma=\left[\left(\omega_{2}-\omega_{1}\right)^{2} /\left(\omega_{2}+\omega_{1}\right)^{2}-\left(\omega_{\text {res }}-\omega_{0}\right)^{2} / \omega_{0}^{2}\right]^{1 / 2}
$$

where $\omega_{1}$ and $\omega_{2}$ are the frequencies of the first band edge mode and third band edge mode, respectively, in the PhC NB structure with $r_{b m}$ obtained from Figure $1 \mathrm{~b} . \omega_{0}$ and $\omega_{\text {res }}$ are the mid-gap frequency $\left(=\left(\omega_{2}+\omega_{1}\right) / 2\right)$ and resonant frequency of the air gap cavity mode, respectively, calculated using the FDTD method. According to the mirror strength, the strongest PBG effect is observed around $\Delta r / r_{0}=0.1$, and therefore, the waveguide loss is minimized. Even though the PBG effect is maximized, the Q-factor is less than 1000, which is not enough for gas sensing. Fortunately, the PBG effect can be strengthened by simply adding mirror air holes. To examine this, we investigated the changes in the $Q$-factor and $S$ as the number of mirror holes increased. For the quantitative analysis and maximization of the Q-factor and $S$ of the air gap cavity mode, we investigated the loss mechanism. As mentioned earlier, the material loss was ignored because Si is a lossless material at a wavelength of $1.55 \mu \mathrm{m}$. Therefore, the total loss, $\alpha_{\text {tot }}$, is caused by the leakage of light, which can be expressed as $\alpha_{\text {tot }}=\alpha_{x}+$ 
$\alpha_{y}+\alpha_{z}$ where $\alpha_{i}$ indicates the optical loss along $i$-axis (the axis direction is shown in Figure 2a). If this equation is re-expressed in terms of the $\mathrm{Q}$-factor, then

$$
1 / \mathrm{Q}_{\mathrm{tot}}=1 / \mathrm{Q}_{x}+1 / \mathrm{Q}_{y}+1 / \mathrm{Q}_{z}
$$

From the simulation, all the Q-factors were obtained by carrying out Poynting flux calculations. Figure $3 \mathrm{c}$ shows that adding more mirror holes reduces the waveguide loss. From these results, we could simultaneously obtain a very high sensitivity $(\sim 1020)$ and a high Q-factor $(>40,000)$ from the TM cavity mode in an optimized PhC NB with an air gap.
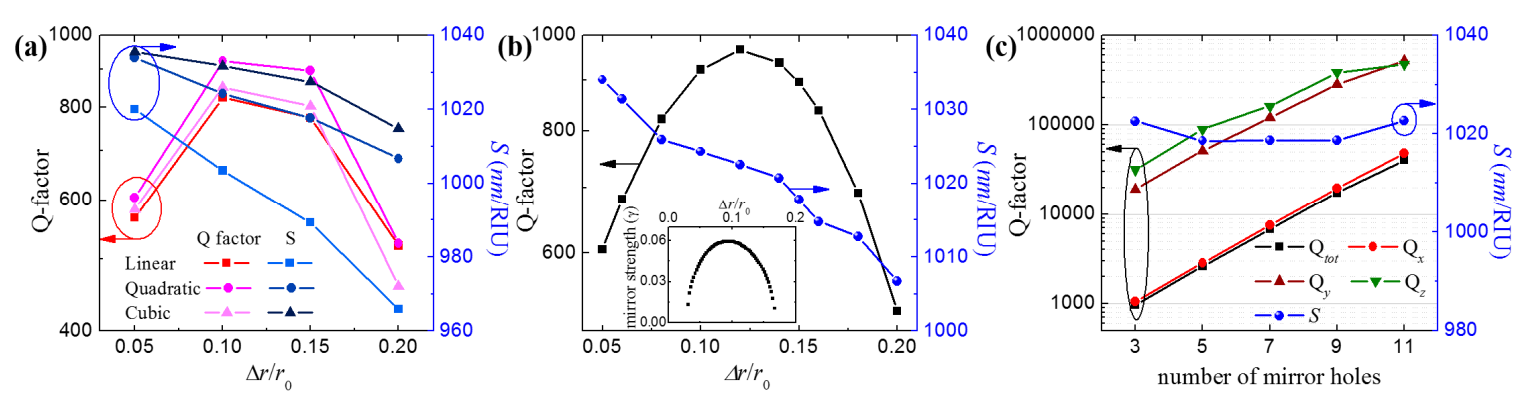

Figure 3. Characteristics of the air gap cavity mode in a PhC NB cavity with a horizontal air gap. (a) Q-factor and $S$ as functions of the variation in the size of air holes with different modulation functions to reduce the air holes from the center to the mirror. In the simulation, $w=0.95 a, t=1.2 a$, $t_{\mathrm{g}}=0.15 a$, and $r_{0}=0.45 a$. (b) Fine optimization of variations in the size of air holes. The inset indicates the mirror strength as a function of variations in the size of air holes. (c) Q-factor and $S$ as functions of the number of mirror holes. In the simulation, all other structural parameters are the same as those in (b) except $\Delta r / r_{0}=0.12$.

As shown in Figure 2d, there is trade-off between the Q-factor and $S$ in the air gap cavity mode in the PhC NB cavity with the horizontal air gap, because a thick horizontal air gap increases the optical sensitivity but decreases the light confinement. Therefore, there is an optimal condition for both a high Q-factor and sensitivity. For the practical design of the optical sensor, we numerically investigated the Q-factor and $S$ of the air gap cavity mode with various thicknesses of the Si waveguide and horizontal air gaps. Figure 4a shows Q-factors of the air gap cavity mode as functions of the thickness of the air gap with various thicknesses of the $\mathrm{Si}$ waveguide. According to these results, the Q-factor increases when the horizontal air gap becomes thin and the Si waveguide becomes thick, which is caused by a strong PBG effect from the high index contrast. However, the value of $S$ of the air gap cavity mode shows exactly the opposite behavior compared with the Q-factor owing to the increase in the air portion (See Figure $4 \mathrm{~b}$ ). In particular, $S$ can exceed $1200 \mathrm{~nm} / \mathrm{RIU}$ when the air portion increases even though the Q-factor becomes low. For the evaluation of the optical sensing performance, the figure of merit (FOM) was defined as follows [26]:

$$
\mathrm{FOM}=S \cdot \mathrm{Q} / \lambda_{\text {res }}
$$

where $\lambda_{\text {res }}$ is the resonant wavelength. Figure $4 \mathrm{c}$ shows the FOM of the air gap cavity mode as a function of thickness of the air gap with various thicknesses of the $\mathrm{Si}$ waveguide. Because the Q-factor increases exponentially with decreasing $t_{\mathrm{g}}$, the FOM shows a similar behavior to that of the Q-factor. For the best performance of the optical sensor, the Q-factor should be balanced with $S$. Therefore, the PhC NB cavity structures with different thicknesses of the Si waveguide have different optimal thicknesses of the air gap, as marked with circles in Figure 4c, which have similar a Q-factor ( 1000) and $S$ ( 1000 $\mathrm{nm} / \mathrm{RIU})$, simultaneously. It is worth noting that by simply increasing the number of mirror holes, the Q-factor could be exponentially increased; therefore, the optimal thickness of the air gap is also changed. 

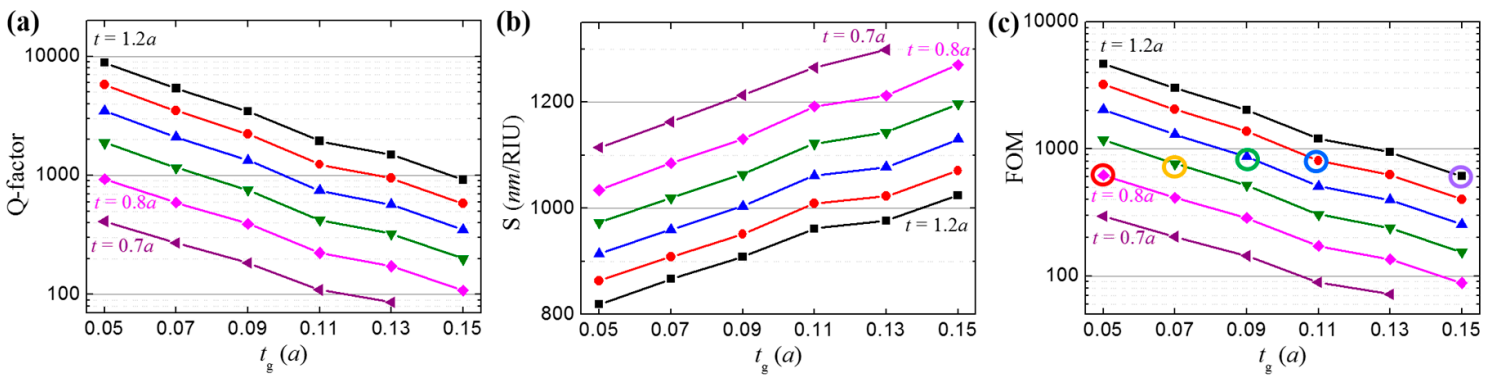

Figure 4. Characteristics of the air gap cavity mode in a PhC NB cavity with various thicknesses $(t)$ of the Si waveguide. (a) Q-factor, (b) S, and (c) figure of merit (FOM) as functions of the thickness of the horizontal air gap $\left(t_{\mathrm{g}}\right)$. In the simulation, the number of mirror holes is three, $w=0.95 a, r_{0}=0.45 a$, and $\Delta r / r_{0}=0.10$. The circles in (c) indicate that the optimal conditions of each $\mathrm{Si}$ waveguide have a similar Q-factor ( 1000) and $S(\sim 1000 \mathrm{~nm} / \mathrm{RIU})$.

\section{Discussion}

In practical applications, it may not be easy to fabricate the air gap using a selective wet etching process due to the capillary-force action $[20,21]$. Therefore, a few parts of the sacrificial layer should be carefully left to avoid the collapse of the dielectric slots by controlling the etching conditions [27]. We studied the effect of the remaining sacrificial layer on optical loss and $S$ when the horizontal etching depth was varied. In the simulation, we used the optimized PhC NB cavity with three mirror holes as shown in Figure 3b. Here, we assume that the etching speed from the sidewall is isotropic. The inset of Figure 5a shows the horizontal cut-view of the sacrificial layer $\left(\mathrm{SiO}_{2}\right)$ at the center of the $\mathrm{PhC} \mathrm{NB}$ cavity structure. The black and gray areas indicate the remaining $\mathrm{SiO}_{2}$ layer and the air, respectively. The white lines indicate the outline of the $\mathrm{Si}$ dielectric slot. When the etching depth exceeds $0.15 a, \mathrm{SiO}_{2}$ at the cavity region is completely removed. Therefore, from the simulation results, both the Q-factor and $S$ are almost constant when the etching depth is over $0.15 a$. However, if the etching depth is less than $0.15 a, S$ decreases suddenly, which is caused by the reduction of the electric field in the environment. When the air switches to $\mathrm{SiO}_{2}$, the mirror strength increases due to the red-shift of the TM band edge modes. Consequently, the waveguide loss decreases and the Q-factor increases. In consideration of mechanical stability and easy fabrication, the $\mathrm{PhC} \mathrm{NB}$ cavity with a $\mathrm{SiO}_{2}$ layer after fine selective etching (depth $<0.05 a$ ) is a good candidate for practical applications of gas sensing because of the high $\mathrm{Q}(>5000)$ and $S(>800)$.
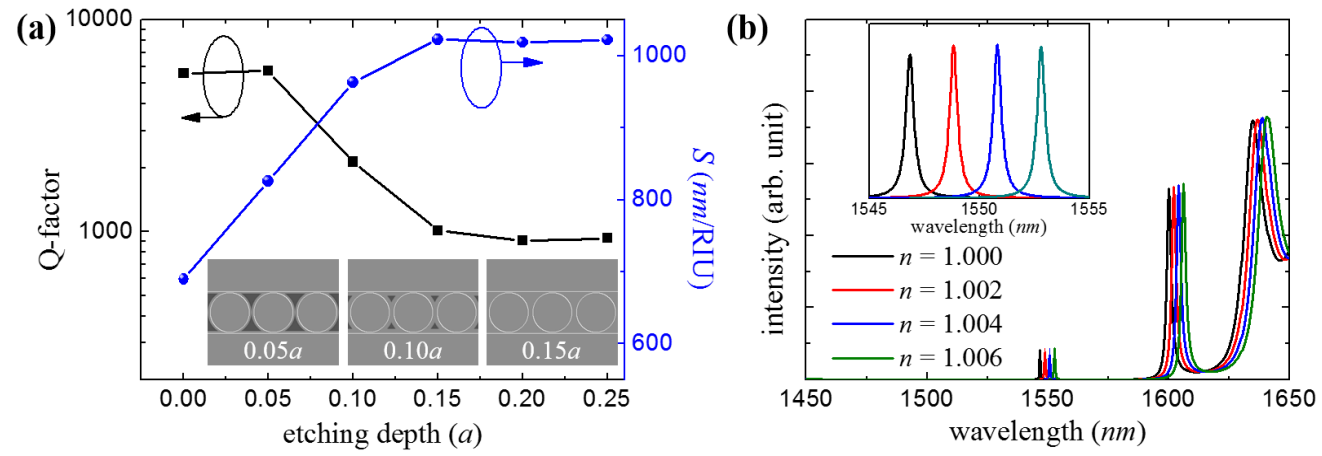

Figure 5. (a) The effect of the remaining sacrificial layer on the Q-factor and $S$ of the air gap mode in the PhC NB cavity as a function of etching depth. The inset shows a horizontal cut-view of the structure with different etching depths. Optimization of the waveguide structure of the PhC NB cavity for refractive index sensing. (b) Transmitted spectra of the PhC NB cavity with different background refractive indices. The inset graph shows the magnified spectra around the resonant wavelength of the air gap mode. 
Finally, we studied the transmission spectra in an Si/air/Si TM waveguide system with the optimized air-slot cavity structure. In the simulation, the total number of mirror holes was set as five in order to save computational time. Figure $5 \mathrm{~b}$ shows the transmission spectra of the PhC NB cavity with different refractive indices of the environment. The sharp peak observed near $1550 \mathrm{~nm}$ corresponds to the TM air gap cavity mode with $a=585 \mathrm{~nm}$. Below $1550 \mathrm{~nm}$, the transmitted intensity is zero owing to the PBG effect. However, modulated peaks are observed beyond $1600 \mathrm{~nm}$. These are the higher order TM air gap cavity modes in the PhC NB cavity. According to the magnified spectra shown in the inset of Figure 5b, the Q-factor and $S$ of the air gap cavity mode are 4000 and $1000 \mathrm{~nm} / \mathrm{RIU}$, respectively. We believe that this high value of $Q$ and ultrasensitive PhC NB TM waveguide system with very small dimensions $\left(\sim 14 \times 0.5 \times 0.8 \mu^{3}\right)$ can be considered promising for compact optical sensors in photonic integrated circuits composed of TM waveguide structures with low index slots filled with dielectric gain media or non-linear materials [19]. In particular, this proposed system is based on the TM guided mode, and therefore can serve as an excellent surface plasmon coupling system with a certain resonance wavelength.

\section{Conclusions}

We numerically investigated a new type of ultrahigh sensitive optical sensor based on the TM mode in a PhC NB cavity structure with a horizontal air gap. The electric field of the resonant TM mode is strongly confined in the horizontal air gap at the center of the dielectric slots of the PhC NB cavity. There is an optimal thickness of the air gap in the dielectric slot because of the trade-off between the Q-factor and $S$ of the TM mode in the PhC NB cavity with an air gap. By optimizing the PhC NB cavity, the maximum sensitivity achieved was over $1000 \mathrm{~nm} / \mathrm{RIU}$ with $\mathrm{Q}>40,000$. When the dielectric slot becomes thin, the sensitivity could be further increased to over $1200 \mathrm{~nm} /$ RIU. For practical applications, a Si-based $\mathrm{PhC}$ NB cavity with a horizontal $\mathrm{SiO}_{2}$ slot structure was proposed, which was found to be mechanically stable after the fine selective etching of $\mathrm{SiO}_{2}$ and maintained high sensitivity with a high Q-factor. This new type of TM resonant mode in PhC NB cavity could be an ideal platform for compact sensors in photonic integrated circuits for TM waveguide systems.

Author Contributions: J.-K.Y.-Supervision, Project administration, Writing-original draft, Writing-Review and Editing, Methodology; C.-Y.K.- Investigation; M.L.-Investigation.

Funding: This research was supported by a research grant from the Kongju National University in 2017.

Conflicts of Interest: The authors declare no conflict of interest.

\section{References}

1. Yablonovitch, E. Inhibited spontaneous emission in solid-state physics and electronics. Phys. Rev. Lett. 1987, 58, 2059-2062. [CrossRef] [PubMed]

2. John, S. Strong localization of photons in certain disordered dielectric lattices. Phys. Rev. Lett. 1987, 58, 2486-2489. [CrossRef] [PubMed]

3. Painter, O.; Lee, R.K.; Yariv, A.; Scherer, A.; O’Brien, J.D.; Dapkus, P.D.; Kim, I. Two-dimensional photonic band-gap defect mode laser. Science 1999, 284, 1819-1824. [CrossRef] [PubMed]

4. Park, H.G.; Kim, S.H.; Kwon, S.H.; Ju, Y.G.; Yang, J.K.; Baek, J.H.; Kim, S.B.; Lee, Y.H. Electrically driven single-cell photonic crystal laser. Science 2004, 305, 1444-1447. [CrossRef] [PubMed]

5. Akahane, Y.; Asano, T.; Song, B.S.; Noda, S. High-Q photonic nanocavity in a two-dimensional photonic crystal. Nature 2003, 425, 944-947. [CrossRef] [PubMed]

6. Lee, K.; Asher, S.A. Photonic crystal chemical sensors: pH and ionic strength. J. Am. Chem. Soc. 2001, 122, 9534-9537. [CrossRef]

7. Loncar, M.; Scherer, A.; Qiu, Y. Photonic crystal laser sources for chemical detection. Appl. Phys. Lett. 2003, 82, 4648-4650. [CrossRef]

8. Choi, J.-H.; No, Y.-S.; So, J.-P.; Lee, J.M.; Kim, K.-H.; Hwang, M.-S.; Kwon, S.-H.; Park, H.-G. A high-resolution strain-gauge nanolaser. Nat. Commun. 2016, 7, 11569. [CrossRef] [PubMed] 
9. Notomi, M.; Kuramochi, E.; Taniyama, H. Ultrahigh-Q nanocavity with 1D photonic gap. Opt. Express 2008, 16, 11095. [CrossRef] [PubMed]

10. Quan, Q.; Deotare, P.B.; Loncar, M. Photonic crystal nanobeam cavity strongly coupled to the feeding waveguide. Appl. Phys. Lett. 2010, 96, 203102. [CrossRef]

11. Jeong, K.Y.; No, Y.S.; Hwang, Y.; Kim, K.S.; Seo, M.K.; Park, H.G.; Lee, Y.H. Electrically driven nanobeam laser. Nat. Commun. 2013, 4, 2822. [CrossRef]

12. Quan, Q.; Burgess, I.B.; Tang, S.K.Y.; Floyd, D.L.; Loncar, M. High-Q, low index-contrast polymeric photonic crystal nanobeam cavities. Opt. Express 2011, 19, 22191-22197. [CrossRef] [PubMed]

13. Kim, S.; Kim, H.-M.; Lee, Y.-H. Single nanobeam optical sensor with a high Q-factor and high sensitivity. Opt. Lett. 2015, 40, 5351. [CrossRef] [PubMed]

14. Almeida, V.R.; Xu, Q.; Barrios, C.A.; Lipson, M. Guiding and confining light in void nanostructure. Opt. Lett. 2004, 29, 1209-1211. [CrossRef] [PubMed]

15. Kwon, S.H.; Sünner, T.; Kamp, M.; Forchel, A. Optimization of photonic crystal cavity for chemical sensing. Opt. Express 2008, 16, 11709-11717. [CrossRef] [PubMed]

16. Kita, S.; Hachuda, S.; Nozaki, K.; Baba, T. Nanoslot laser. Appl. Phys. Lett. 2010, 97, 161108. [CrossRef]

17. Wang, B.W.; Dundar, M.A.; Notzel, R.; Karouta, F.; He, S.L.; van der Heijden, R.W. Photonic crystal slot nanobeam slow light waveguides for refractive index sensing. Appl. Phys. Lett. 2010, 97, 151105. [CrossRef]

18. Yang, D.; Zhang, P.; Tian, H.; Ji, Y.; Quan, Q. Ultrahigh-Q and low mode volume parabolic radius-modulated single photonic crystal slot nanobeam cavity for high-sensitive refractive index sensing. IEEE Photonics J. 2015, 7, 4501408. [CrossRef]

19. Sun, R.; Dong, P.; Feng, N.N.; Hong, C.Y.; Michel, J.; Lipson, M.; Kimerling, L. Horizontal single and multiple slot waveguides: Optical transmission at $\lambda=1550 \mathrm{~nm}$. Opt. Express 2007, 15, 17967-17972. [CrossRef] [PubMed]

20. Lee, S.; Eom, S.; Chang, J.; Huh, C.; Sung, G.; Shin, J. A silicon nitride microdisk resonator with a 40-nm-thin horizontal air slot. Opt. Express 2010, 18, 11209-11215. [CrossRef] [PubMed]

21. Jang, H.; Karnadi, I.; Pramudita, P.; Song, J.-H.; Kim, K.S.; Lee, Y.-H. Sub-microWatt threshold nanoisland lasers. Nat. Commun. 2015, 6, 8276. [CrossRef] [PubMed]

22. Zhang, Y.; McCutcheon, M.W.; Burgess, I.B.; Loncar, M. Ultra-high-Q TE/TM dual-polarized photonic crystal nanocavities. Opt. Lett. 2009, 34, 2694-2696. [CrossRef] [PubMed]

23. Lu, T.-W.; Lin, P.-T.; Lee, P.-T. Photonic crystal horizontally slotted nanobeam cavity for silicon-based nanolasers. Opt. Lett. 2012, 37, 569-571. [CrossRef] [PubMed]

24. Lu, T.-W.; Lin, P.-T.; Lee, P.-T. One-dimensional photonic crystal fishbone hybrid nanocavity with nanoposts. Appl. Phys. Lett. 2014, 104, 191107. [CrossRef]

25. Johnson, S.G.; Joannopoulos, J. Block-iterative frequency domain methods for Maxwell's equations in a planewave basis. Opt. Express 2001, 8, 173. [CrossRef] [PubMed]

26. Qiao, Q.; Xia, J.; Lee, C.; Zhou, G. Application of photonic crystal nanobeam cavities for sensing. Micromachines 2018, 9, 541. [CrossRef] [PubMed]

27. Choi, J.-H.; No, Y.-S.; Hwang, M.-S.; Kwon, S.-Y.; Jeong, K.-Y.; Kwon, S.-H.; Yang, J.-K.; Park, H.-G. Low-threshold photonic-band-edge laser using iron-nail-shaped rod array. Appl. Phys. Lett. 2014, 104, 091120. [CrossRef]

(C) 2019 by the authors. Licensee MDPI, Basel, Switzerland. This article is an open access article distributed under the terms and conditions of the Creative Commons Attribution (CC BY) license (http://creativecommons.org/licenses/by/4.0/). 